\title{
Intima media thickness and brachial artery flow mediated dilatation in women with polycystic ovary syndrome and type 1 diabetes mellitus
}

Agnieszka Łebkowska ${ }^{1}$, Agnieszka Adamska ${ }^{1}$, Joanna Tołwińska ${ }^{2}$, Małgorzata Jacewicz $^{1}$, Justyna Hryniewicka ${ }^{1}$, Robert Milewski ${ }^{3}$, Sławomir Wołczyński ${ }^{4}$, Artur Bossowski², Maria Górska', Irina Kowalska ${ }^{1}$

${ }^{1}$ Department of Endocrinology, Diabetology and Internal Medicine, Medical University of Bialystok, Poland

2Department of Pediatrics, Endocrinology, Diabetology with Cardiology Division, Medical University of Bialystok, Poland

${ }^{3}$ Department of Statistics and Medical Informatics, Medical University of Bialystok, Poland

${ }^{4}$ Department of Reproduction and Gynecological Endocrinology, Medical University of Bialystok, Poland

\section{Background}

Polycystic ovary syndrome (PCOS) and type 1 diabetes (T1DM) are accompanied by increased risk of atherosclerosis. A higher prevalence of PCOS in T1DM patients has been reported, which could predispose this group of patients to higher risk of cardiovascular disease. Brachial artery flow-mediated dilatation (FMD) and intima media thickness of common carotid artery (IMT) are used to detect an early endothelial dysfunction

\section{Aim}

The aim of our study was to examine IMT and FMD in T1DM women with PCOS. We also estimated the relation of IMT and FMD to clinical and hormonal parameters in the studied population

\section{Subjects and methods}

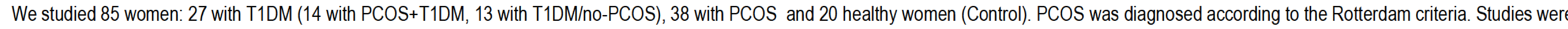

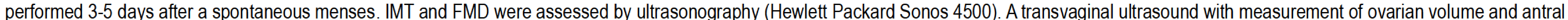

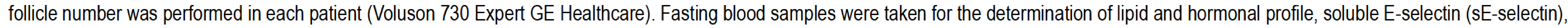

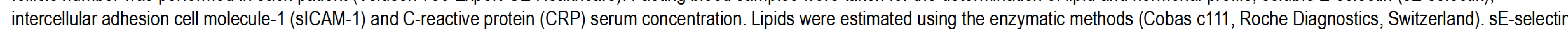

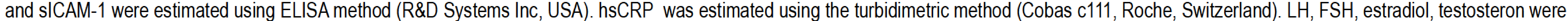
measured using the RIA method (DiaSource, Belgium)

\section{Results}

Table 1. Clinical characteristics of the studied groups

\begin{tabular}{|c|c|c|c|c|}
\hline & PCOS+T1DM (n=14) & $\begin{array}{l}\text { PCOS } \\
(n=38)\end{array}$ & $\begin{array}{l}\text { T1DM/no-PCOS } \\
(n=13)\end{array}$ & $\begin{array}{l}\text { Control } \\
(n=20)\end{array}$ \\
\hline Age (yr) & $\begin{array}{l}24.0 \\
(21.0-28.0)\end{array}$ & $\begin{array}{l}24.0 \\
(23.0-27.0)\end{array}$ & $\begin{array}{l}27.0 \\
(21.0-32.0)\end{array}$ & $\begin{array}{l}23.5 \\
(22.0-26.5)\end{array}$ \\
\hline BMI $\left(\mathrm{kg} / \mathrm{m}^{2}\right)$ & $\begin{array}{l}25.4 \\
(22.2-26.4)\end{array}$ & $\begin{array}{l}25.51 \\
(21.8-30.0)\end{array}$ & $\begin{array}{l}24.4 \\
(23.5-27.7)\end{array}$ & $\begin{array}{l}21.7 \\
(20.2-23.3)\end{array}$ \\
\hline DM duration (yr) & $\begin{array}{l}10 \\
(8-13)\end{array}$ & & $\begin{array}{l}12 \\
(4-16)\end{array}$ & \\
\hline HbA1c (\%) & $\begin{array}{l}6.65 \\
(6.50-7.60)\end{array}$ & & $\begin{array}{l}8.10 \\
(7.10-8.80)\end{array}$ & \\
\hline Insulin dose (U/24h) & $\begin{array}{l}34.5 \\
(30.0-48.0)\end{array}$ & & $\begin{array}{l}46.0 \\
(27.0-55.0)\end{array}$ & \\
\hline Ovarian volume ( $\mathrm{ml}$ ) & $\begin{array}{l}13.0 \\
(10.7-20.3)\end{array}$ & $\begin{array}{l}15.5 \\
(10.5-19.0)\end{array}$ & $\begin{array}{l}9.5 \\
(9.3-10.2)\end{array}$ & $\begin{array}{l}10.9 \\
(8.1-15.0)\end{array}$ \\
\hline $\begin{array}{l}\text { Ovarian follicle } \\
\text { number }\end{array}$ & $\begin{array}{l}19^{2} \\
(18-29)\end{array}$ & $\begin{array}{l}24^{1} \\
(18-34)\end{array}$ & $\begin{array}{l}14 \\
(10-16)\end{array}$ & $\begin{array}{l}14 \\
(12-17)\end{array}$ \\
\hline sE-selectin (ng/ml) & $\begin{array}{l}38.1^{3} \\
(26.7-52.0)\end{array}$ & $\begin{array}{l}23.8 \\
(15.7-37.3)\end{array}$ & $\begin{array}{l}35.9 \\
(34.4-44.6)\end{array}$ & $\begin{array}{l}28.1 \\
(16.8-35.1)\end{array}$ \\
\hline sICAM-1 (ng/ml) & $\begin{array}{l}256.1^{3} \\
(200.8-291.6)\end{array}$ & $\begin{array}{l}209.7 \\
(169.9-232.9)\end{array}$ & $\begin{array}{l}261.1 \\
(226.3-257.9)\end{array}$ & $\begin{array}{l}219.8 \\
(179.7-221.4\end{array}$ \\
\hline
\end{tabular}

Figure 1. IMT and FMD in the studied groups
Table 2. Hormonal and lipid profile of the studied groups

\begin{tabular}{|c|c|c|c|c|}
\hline & PCOS+T1DM $(n=14)$ & $\begin{array}{l}\text { PCOS } \\
(n=38)\end{array}$ & $\begin{array}{l}\text { T1DM/no- PCOS } \\
(n=13)\end{array}$ & $\begin{array}{l}\text { Control } \\
(\mathrm{n}=20)\end{array}$ \\
\hline LH (mlU/ml) & $\begin{array}{l}4.23 \\
(3.35-6.02)\end{array}$ & $\begin{array}{l}4.60 \\
(3.54-6.00)\end{array}$ & $\begin{array}{l}3.47 \\
(2.23-4.28)\end{array}$ & $\begin{array}{l}3.86 \\
(2.82-4.36)\end{array}$ \\
\hline FSH (mlU/ml) & $\begin{array}{l}4.44 \\
(3.53-5.88)\end{array}$ & $\begin{array}{l}4.17 \\
(3.00-5.37)\end{array}$ & $\begin{array}{l}4.72 \\
(3.62-5.51)\end{array}$ & $\begin{array}{l}5.75 \\
(5.11-8.10)\end{array}$ \\
\hline Estradiol (pg/ml) & $\begin{array}{l}51.71 \\
(37.00-72.00)\end{array}$ & $\begin{array}{l}77.00 \\
(46.00-131.25)\end{array}$ & $\begin{array}{l}89.53 \\
(60.52-150.87)\end{array}$ & $\begin{array}{l}68.01 \\
(45.00-80.00)\end{array}$ \\
\hline Testosterone (ng/ml) & $\begin{array}{l}0.69 \\
(0.55-1.06)\end{array}$ & $\begin{array}{l}0.88^{1} \\
(0.59-1.12)\end{array}$ & $\begin{array}{l}0.53 \\
(0.43-0.72)\end{array}$ & $\begin{array}{l}0.51 \\
(0.39-0.70)\end{array}$ \\
\hline $\begin{array}{l}\text { Total Cholesterol } \\
\text { (mg/dl) }\end{array}$ & $\begin{array}{l}169.5 \\
(160.0-176.0)\end{array}$ & $\begin{array}{l}181.0 \\
(161.0-201.0)\end{array}$ & $\begin{array}{l}171.0 \\
(137.0-189.0)\end{array}$ & $\begin{array}{l}165.5 \\
(154.0-180.5)\end{array}$ \\
\hline LDL (mg/dl) & $\begin{array}{l}90.8 \\
(80.4-99.2)\end{array}$ & $\begin{array}{l}100.1 \\
(81.8-119.6)\end{array}$ & $\begin{array}{l}90.4 \\
(55.6-95.4)\end{array}$ & $\begin{array}{l}88.5 \\
(68.6-104.8)\end{array}$ \\
\hline $\begin{array}{l}\text { Triglycerides } \\
\text { (mg/dl) }\end{array}$ & $\begin{array}{l}58.5 \\
(52.0-69.0)\end{array}$ & $\begin{array}{l}65.5 \\
(51.0-106.0)\end{array}$ & $\begin{array}{l}79.0 \\
(64.0-108.0)\end{array}$ & $\begin{array}{l}59.0 \\
(39.5-81.0)\end{array}$ \\
\hline HDL (mg/dl) & $\begin{array}{l}65.0 \\
(50.0-72.0)\end{array}$ & $\begin{array}{l}61.0 \\
(51.0-78.0)\end{array}$ & $\begin{array}{l}64.0 \\
(57.0-81.0)\end{array}$ & $\begin{array}{l}65.5 \\
(59.5-78.0)\end{array}$ \\
\hline hsCRP (mg/l) & $\begin{array}{l}1.65 \\
(0.34-3.06)\end{array}$ & $\begin{array}{l}0.57 \\
(0.12-1.92)\end{array}$ & $\begin{array}{l}1.40 \\
(0.70-2.10)\end{array}$ & $\begin{array}{l}0.28 \\
(0.12-1.22)\end{array}$ \\
\hline
\end{tabular}

Figure 2. Correlation of IMT and triglycerides in PCOS+T1DM

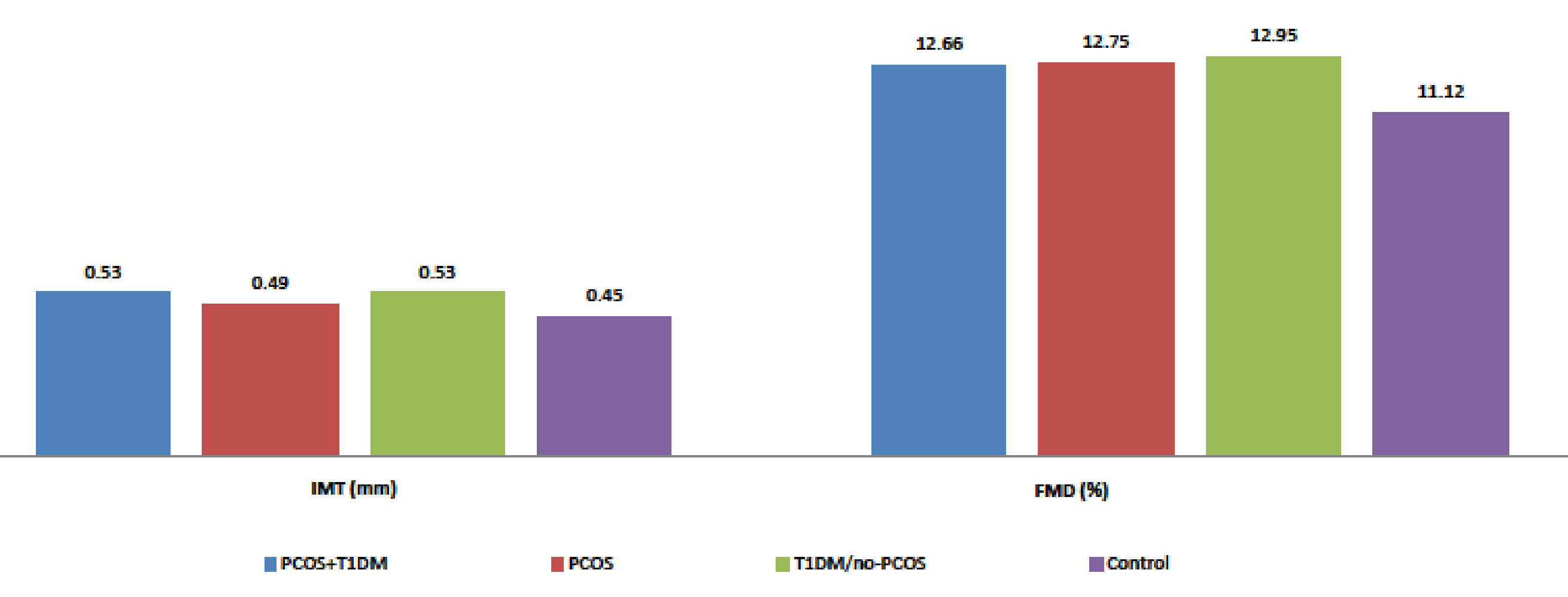

Table 1. Correlations of IMT in the entire studied group

\begin{tabular}{|l|l|l|}
\hline \multicolumn{1}{|c|}{} & IMT & \\
\hline & $\mathbf{r}$ & $\mathbf{p}$ \\
\hline BMI & 0.36 & 0.001 \\
\hline CRP & 0.26 & 0.044 \\
\hline sE-selectin & 0.23 & 0.033 \\
\hline sICAM-1 & 0.29 & 0.007 \\
\hline
\end{tabular}

Table 2. Correlations of IMT and FMD in T1DM

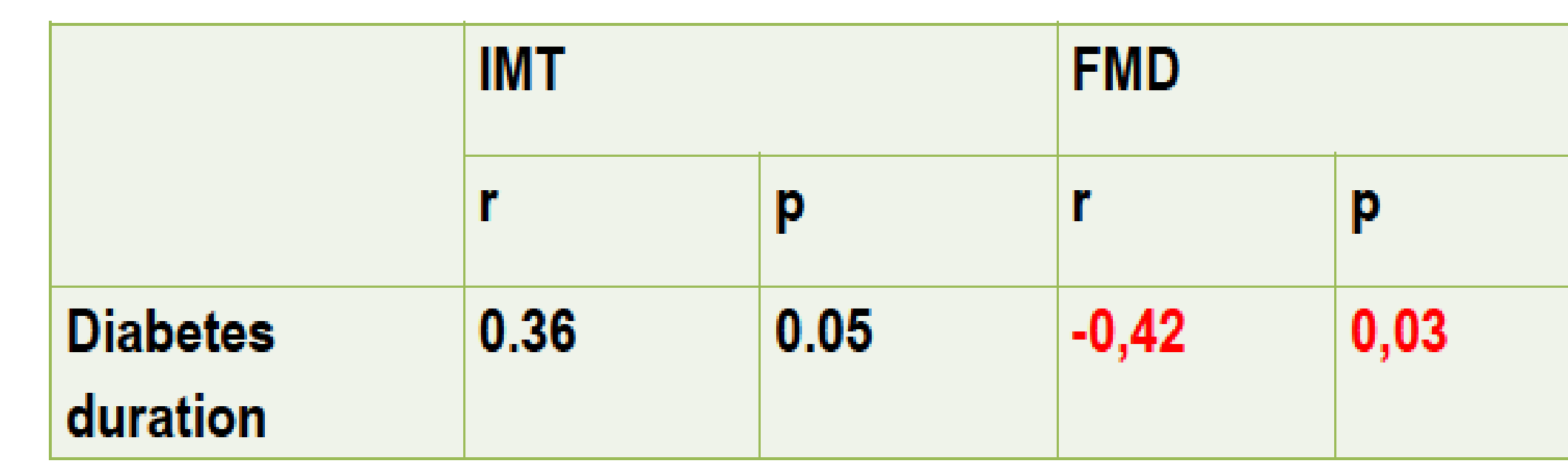

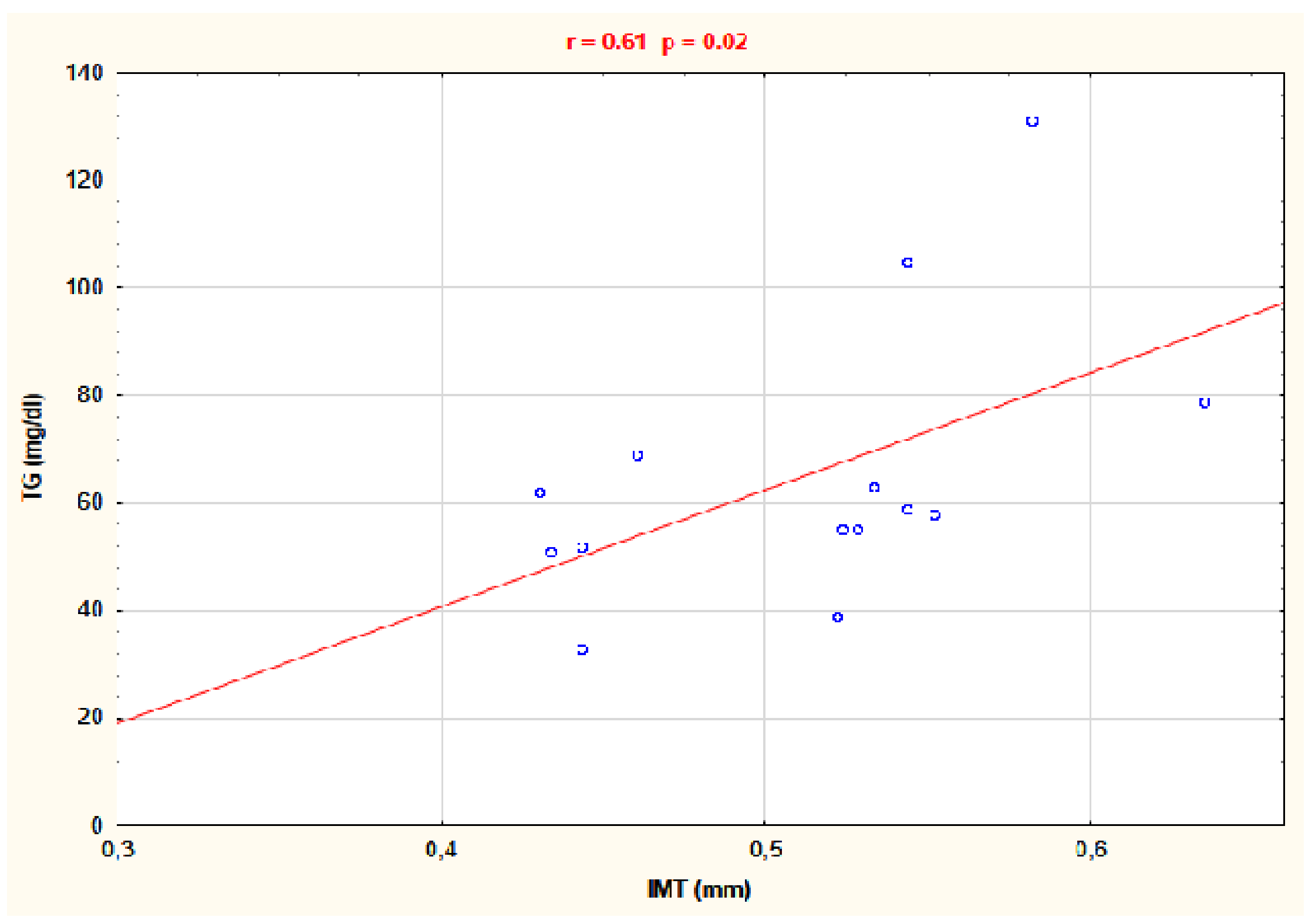

\section{Conclusions}

Our data suggest that early vascular changes in young T1DM patients are related to diabetes duration and additionally, in patients with PCOS+T1DM, to atherogenic lipid profile. 